\title{
LAS CURTIEMBRES DE EL CERRITO (VALLE DEL CAUCA): UNA MIRADA EN TORNO A SU REALIDAD SOCIOECONOMICA Y AMBIENTAL
}

\section{TANNERIES OF EL CERRITO (VALLE DEL CAUCA): A LOOK ABOUT YOUR ENVIRONMENTAL AND SOCIOECONOMIC REALITY}

\author{
Neyla Benítez-Campo ${ }^{1}$, Aceneth Perafán Cabrera²
}

\begin{abstract}
${ }^{1}$ Bióloga, M.Sc. Microbiología, Ph.D. Ciencias Ambientales, Grupo de investigación en Biología de Plantas y Microorganismos. Universidad del Valle, Calle 13 No. 100-00, Cali, Colombia, e-mail: neyla.benitez@correounivalle.edu.co; ${ }^{2}$ Licenciada en Ciencias Sociales, Ph.D. Medio ambiente natural y humano en las ciencias sociales, Grupo de investigación Asociación Centro de estudios regionales, REGIÓN. Universidad del Valle, Calle 13 No. 100-00, Cali, Colombia, e-mail: acenethperafan@ hotmail.com
\end{abstract}

Rev. U.D.C.A Act. \& Div. Cient. 19(2): 457-466, Julio-Diciembre, 2016

\section{RESUMEN}

El curtido de pieles es un proceso reconocido por el vertimiento de desechos químicos y orgánicos, así como por la liberación de compuestos volátiles de olores desagradables y desechos sólidos, que contaminan el agua, el aire y el suelo, produciendo efectos negativos en el ambiente natural y en la salud de la población. Considerando que este comportamiento está influenciado por componentes interrelacionados, el propósito de esta investigación fue identificar los principales factores socioeconómicos y ambientales que caracterizan la actividad curtidora en el municipio de El Cerrito, Valle del Cauca. Mediante el empleo de métodos cuantitativos y cualitativos, que incluyeron entrevistas con actores sociales clave, revisión bibliográfica y trabajo de campo, se pudo establecer que de 13 curtiembres activas, una de ellas corresponde a mediana empresa y las 12 restantes a microempresas (McEs), con cerca de 272 empleados directos. Dichas empresas han diversificado su actividad, dedicándose al curtido de pieles y al alquiler de servicios a curtidores informales. Las McEs presentan una baja capacidad de inversión en maquinaria y en equipos, en modernización tecnológica y en la adopción de programas e infraestructura de mejoramiento ambiental. Se evidenció la falta de conciencia ambiental, de planes de capacitación, de acompañamiento técnico y de políticas gubernamentales que promuevan su desarrollo y la adopción efectiva de programas de control y de prevención de la contaminación. Las características de este sector industrial hacen necesaria la implementación de programas integrales y adaptables a las McEs, de tal manera que conduzcan a mejorar su competitividad y sostenibilidad.
Palabras clave: Contaminación, cromo, cuero, producción más limpia, sostenibilidad.

\section{SUMMARY}

Tanning is a process recognized for the dumping of chemical and organic wastes, as well as the release of volatile compounds of unpleasant odors and solid wastes that pollute water, air and soil, producing negative effects on the environment and the health of the population. Since this behavior is influenced by interrelated components, the purpose of this research was to identify the main socioeconomic and environmental factors that characterize the tanning activity in the municipality of El Cerrito, Valle del Cauca. By using quantitative and qualitative methods, which included interviews with key social actors, literature review and fieldwork, it was established that 13 active tanneries, one corresponding to amedium enterprise and the remaining 12 to microenterprises (ECMs) with about 272 direct employees. These companies have diversified their activities, dedicated to tanning and rental services to informal tanners. ECMs have a low capacity for investment in machinery and equipment, technological modernization and the adoption of environmental programs and infrastructure improvement. The lack of environmental awareness, training plans, technical support and government policies that promote the development and adoption of effective control programs and pollution prevention was evident. The characteristics of this industry require the implementation of comprehensive and adaptable programs for ECMs, so leading to improve their competitiveness and sustainability. 
Key words: Pollution, chrome, leather, cleaner production, sustainability.

\section{INTRODUCCIÓN}

Las curtiembres, se dedican al curtido de pieles de vacunos, de ovinos y de caprinos para convertirlas en cuero, material duradero, apenas permeable al agua y, a la vez, suave, elástico y flexible (Lofrano et al. 2013). El curtido es un método ancestral que estabiliza la materia orgánica, inhibiendo sus procesos de descomposición, mediante una serie de etapas, en las que es necesario adicionar productos químicos, que tienen consecuencias ambientales significativas, especialmente, por los grandes volúmenes de agua con desechos orgánicos y químicos, que son vertidos a los cuerpos de agua (Faouzi et al. 2013; llou et al. 2014).

Esta actividad industrial es, además, altamente demandante de recursos naturales, por cada $1000 \mathrm{~kg}$ de piel salada que entran al proceso, se requieren de 15 a $40 \mathrm{~m}^{3}$ de agua y $450 \mathrm{~kg}$ de insumos químicos. Se obtienen $200 \mathrm{~kg}$ de cuero acabado, $40 \mathrm{~kg}$ de solventes que son emitidos a la atmósfera, $640 \mathrm{~kg}$ de residuos sólidos y $138 \mathrm{~L}$ de agua que pierde la piel (Centro Nacional de Producción Más Limpia, 2004a).

Es evidente que, desde el punto de vista ambiental, las curtiembres generan contaminantes derivados del aprovechamiento que hacen de la piel, un subproducto altamente putrescible y de degradación lenta de la cadena del cuero. A su vez, el cuero es distribuido por peleterías a industrias manufactureras, que lo transforman en calzado, en guantes, en ropa, en artículos de marroquinería, en muebles y en otros productos artesanales (Ministerio de Ambiente Vivienda y Desarrollo Territorial, 2006).

Se han realizado estudios sobre el impacto ambiental de las curtiembres, la baja infraestructura y mínima capacidad productiva en países en desarrollo (Centro de Promoción de Tecnologías Sostenibles 2009; Centro Nacional de Producción Más Limpia, 2004b; Ministerio de Ambiente Vivienda y desarrollo territorial, 2006; Azom et al. 2012; Shakir et al. 2012). Adicionalmente, se ha informado de avances en procesos de reutilización y de reciclaje de efluentes, para disminución de la carga contaminante y ahorros de agua, así como recuperación de residuos al aplicar programas de Producción Más Limpia (PML), con beneficios económicos, en tenerías europeas y latinoamericanas (Ministerio de Ambiente Vivienda y Desarrollo Territorial, 2006; Centro de Promoción de Tecnologías Sostenibles, 2009; COTANCE \& IndustriAll, 2012).

En las curtiembres de Villapinzón y de Chocontá, se han desarrollado proyectos con el propósito de mejorar el desempeño ambiental del curtido de pieles, manejo de subproductos y comercialización de cueros, propendiendo por construir escenarios de mejoramiento de la calidad del agua del río Bogotá y de la industria curtidora de la región (Corporación Autónoma Regional de Cundinamarca. Universidad Nacional de Colombia, 2009).

De igual manera, la Corporación Autónoma Regional del Valle del Cauca (CVC) y el Centro Regional de Producción Más Limpia (CRPML) realizaron un proyecto de PML y gestión ambiental con las curtiembres del municipio de El Cerrito (Jaramillo et al. 2005a). Posteriormente, el CRPML, el instituto CINARA de la Universidad del Valle y la Corporación de Desarrollo Productivo del Cuero, Calzado y Marroquinería (CDP) continuaron apoyando al sector, a través de proyectos que permitieron difundir, en algunos empresarios, los principios de prevención de la contaminación, a través del concepto de PML, para el mejoramiento ambiental (Restrepo et al. 2006).

Por otro lado, se han realizado algunos estudios con un enfoque integral del problema de contaminación de las curtiembres, en los que se reconocen los posibles beneficios biofísicos, socioculturales y económicos de las estrategias de PML con inclusión social, en conjunto con los aspectos legales y la gestión interinstitucional para su aplicación exitosa, en las tenerías de Villapinzón y Chocontá, en Cundinamarca (Santos et al. 2007; Sanz et al. 2016); sin embargo, estos programas siguen en proceso de adaptación y mejora. En algunos casos, se lograron avances, que no se han podido mantener en el tiempo, razones que justifican la falta de conocimiento acerca de las dinámicas que se mueven al interior de este sector industrial, de tal manera que permitan investigar, desarrollar y aplicar nuevos métodos, en busca de una mayor sostenibilidad de las curtiembres.

Teniendo en cuenta un enfoque integral de la problemática de contaminación de las curtiembres, el propósito de este trabajo fue identificar los principales factores socioeconómicos y ambientales que caracterizan la actividad curtidora, en el municipio de El Cerrito, en el Valle del Cauca.

\section{MATERIALES Y MÉTODOS}

Este estudio, se realizó en el municipio de El Cerrito, ubicado en el departamento del Valle del Cauca - Colombia (Figura 1), a una altura de $987 \mathrm{msnm}$, temperatura promedio de $24^{\circ} \mathrm{C}$, con una población aproximada de 57.463 habitantes. El sector de las curtiembres es considerado el tercer renglón económico del municipio, después de la caña de azúcar y la ganadería (Alcaldía de El Cerrito Valle del Cauca, 2013).

La población, objeto de estudio, estuvo representada por empresarios y trabajadores de las curtiembres; adicionalmente, se incluyeron en la muestra, a aquellos funcionarios que, por sus cargos, pudieran tener relación con dicho sec- 


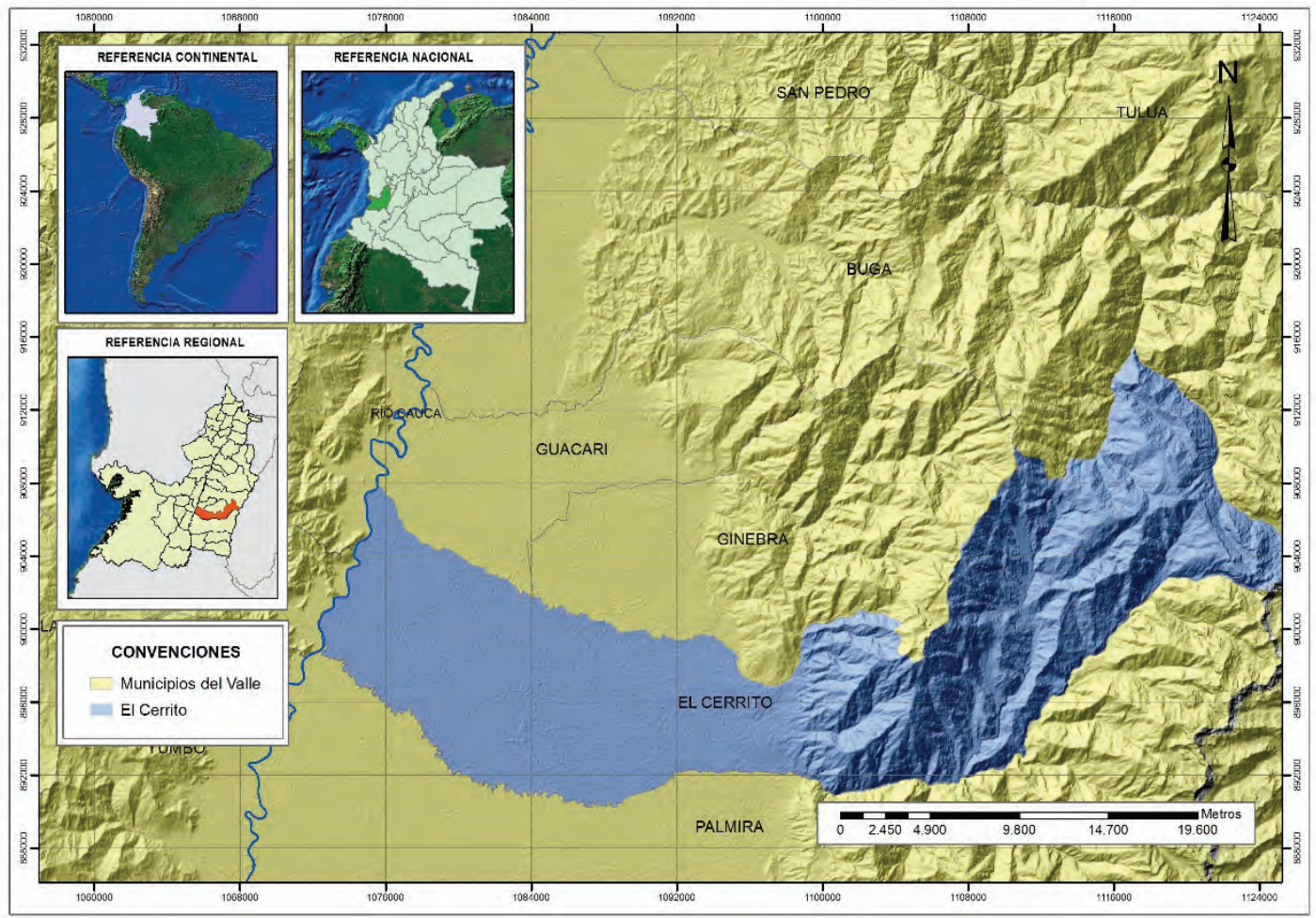

Figura 1. Ubicación geográfica del municipio de El Cerrito.

tor, tales como empleados de la Secretaría de Salud municipal, de la Oficina de Planeación Municipal, de la Corporación Autónoma Regional del Valle del Cauca (CVC) y la Sociedad de Acueductos y Alcantarillados del Valle del Cauca S.A. (ACUAVALLE S.A.). De manera complementaria, se identificaron algunos miembros de la comunidad aledaña a las curtiembres, que por su cercanía podían aportar información valiosa, para los propósitos de la investigación.

La recolección de la información, se realizó entre enero de 2013 y octubre de 2014, para lo cual, se diseñaron y aplicaron entrevistas semiestructuradas, específicas para cada tipo de actor social. En cuanto a los empresarios, se obtuvo información en relación a los orígenes de la curtiembre, los aspectos productivos, económicos y socio-ambientales; para el caso de los trabajadores, se indagó acerca de las condiciones laborales, seguridad social y salud, proceso productivo y aspectos socio-ambientales y, en lo que respecta a los funcionarios, las preguntas buscaron identificar su relación con las curtiembres y aspectos socio-ambientales. Esta información, se complementó con los datos reportados por los vecinos en cuanto a las enfermedades frecuentes, aspectos socio-económicos y socio-ambientales de las curtiembres (Tabla 1).
En total, se entrevistaron 23 mujeres y 31 hombres, mayores de 30 años correspondientes a población laboralmente activa en un $94 \%$ y seleccionados según el rol de interés dentro de este estudio. La información obtenida fue organizada en tablas y gráficas y posteriormente, se calcularon frecuencias relativas y medidas de tendencia central. Con el fin de analizar la relación entre algunas variables cualitativas, se utilizó la prueba exacta de Fisher, procesamiento estadístico que se realizó mediante el software $\mathrm{R}$ versión 3.2.2 (R Core Team, 2015) con un nivel de significancia del $5 \%$.

\section{RESULTADOS Y DISCUSIÓN}

En Colombia, el comportamiento del sector del cuero y sus manufacturas en los últimos años, ha mostrado tendencia a la baja y varía según el eslabón de la cadena. Durante el 2013, la actividad manufacturera de curtido y preparado de cueros, productos de cuero y calzado, decreció un 5,7\% con respecto a 2012, muy por debajo del crecimiento del país (Departamento Administrativo Nacional de Estadística, 2014); la participación de este sector en la economía nacional estuvo alrededor del 0,27\%, del PIB nacional y $2,17 \%$, del PIB manufacturero (Aktiva, 2013). 
Tabla 1. Estructura del cuestionario.

\begin{tabular}{|c|c|}
\hline ACTOR SOCIAL & TIPO DE PREGUNTA \\
\hline \multirow[t]{4}{*}{ Trabajadores (18) } & Datos generales: Nombre, edad, sexo, nivel educativo, formación en curtiembres. \\
\hline & $\begin{array}{l}\text { Información laboral: Cargo y funciones, tiempo de trabajo, horario laboral, remuneración, proce- } \\
\text { so productivo, Estado actual del sector de las curtiembres. }\end{array}$ \\
\hline & $\begin{array}{l}\text { Seguridad social y salud: EPS, Fondo de pensiones, implementos de protección laboral, recibe } \\
\text { capacitación, existen programas de salud ocupacional, enfermedades frecuentes. }\end{array}$ \\
\hline & $\begin{array}{l}\text { Aspectos socio-ambientales: Riesgos del uso del Cr, programas de GA, PML, posee PTAR, di- } \\
\text { ficultades para implementar soluciones a la contaminación, relación con la CVC y autoridades } \\
\text { municipales, estado del río Cerrito. }\end{array}$ \\
\hline \multirow[t]{4}{*}{ Empresarios (13) } & $\begin{array}{l}\text { Datos generales: Nombre, edad, sexo, nivel educativo, formación en curtiembres, tiempo de tra- } \\
\text { bajo, heredero. }\end{array}$ \\
\hline & Información de la curtiembre: Nombre, año de creación, actividad principal \\
\hline & $\begin{array}{l}\text { Aspectos productivos y económicos: Principales productos, cuales servicios presta, principales } \\
\text { clientes, origen y tamaño de las pieles, No. pieles/mes, Vr/piel, en que se usan sus productos, pro- } \\
\text { piedad del local, área del local, No. de Bombos y de máquinas. Procesos realizados dentro y fuera } \\
\text { de la curtiembre. No. de trabajadores, No. de hombres, No de mujeres, cuantos administrativos, } \\
\text { cuantos operarios, nivel educativo de los trabajadores, tipo de contrato, tipo de seguridad social, } \\
\text { Estado actual del sector de las curtiembres. }\end{array}$ \\
\hline & $\begin{array}{l}\text { Aspectos socio-ambientales: Fuentes de energía, Fuentes de agua, consumo mensual. Posee } \\
\text { programa de residuos sólidos, Que hacen con las Aguas residuales, tipo de PTAR, Programa de } \\
\text { PML, Beneficios de PML, Riesgos del Cr., dificultades para implementar soluciones, reciben aseso- } \\
\text { ría de alguna entidad, relación con la CVC y autoridades municipales, quejas de los vecinos, estado } \\
\text { del río Cerrito. }\end{array}$ \\
\hline \multirow{3}{*}{$\begin{array}{l}\text { Funcionarios CVC } \\
(2) \\
\text { Salud (1) } \\
\text { Alcaldía (2) } \\
\text { ACUAVALLE (2) }\end{array}$} & $\begin{array}{l}\text { Datos generales: Nombre, edad, sexo, formación académica, cargo y funciones, tiempo en el } \\
\text { cargo. }\end{array}$ \\
\hline & Relación con las curtiembres: Programas propuestos para las curtiembres \\
\hline & $\begin{array}{l}\text { Aspectos socio-ambientales: Problemas más relevantes de las curtiembres, quejas de la comuni- } \\
\text { dad, Participación ciudadana, conoce proyectos exitosos en las curtiembres, actividades realizadas } \\
\text { para el manejo del agua residual, dificultades para implementar soluciones, que entidades han } \\
\text { realizado control y prevención dela contaminación en curtiembres, Estado actual del sector de las } \\
\text { curtiembres. Estado del río Cerrito. }\end{array}$ \\
\hline \multirow[t]{4}{*}{ Vecinos (16) } & Datos generales: Nombre, edad, sexo, nivel educativo, ocupación, tiempo de residencia. \\
\hline & Salud: Enfermedades frecuentes, posee EPS. \\
\hline & Aspectos socioeconómicos. Fuentes de empleo, percepción sobre las curtiembres. \\
\hline & $\begin{array}{l}\text { Aspectos socio-ambientales: Calidad del servicio de acueducto, recolección de basuras, princi- } \\
\text { pales problemas ambientales, han percibido cambios en el paisaje, posibles causas de las trans- } \\
\text { formaciones, participación comunitaria en la solución de los problemas, acciones institucionales, } \\
\text { estado del río Cerrito }\end{array}$ \\
\hline
\end{tabular}

GA: Gestión ambiental, PML: Producción Más Limpia, PTAR: Planta de Tratamiento de Aguas Residuales. Entre paréntesis se indica el número de entrevistados. 
Sucesos como el ritmo negativo de crecimiento del sector del cuero y sus manufacturas, a nivel mundial, debido a la decadencia del consumo de cuero y a la fuerte competencia de materiales sintéticos, así como al aumento de las importaciones de productos de cuero provenientes de países asiáticos, como consecuencia de políticas de apertura económica, influyeron de manera negativa en este sector manufacturero (Restrepo et al. 2006).

La situación de las curtiembres de El Cerrito no escapa al contexto nacional, puesto que se encontraron funcionando sólo 13 curtiembres, lo que representa una disminución del $38,1 \%$ al comparar esta información con la obtenida por Jaramillo et al. (2005b), quienes habían reportado 21 curtiembres activas en ese año.

Esta comunidad empresarial está compuesta por una mediana empresa y 12 microempresas (McEs), que generan alrededor de 272 empleos directos, observándose una disminución del $28,4 \%$, en comparación con los datos obtenidos por Jaramillo et al. (2005b). De igual manera, los entrevistados coincidieron en afirmar que han bajado la producción entre un 50 a $60 \%$ de su capacidad, debido a la escasez de pieles de buena calidad, que son exportadas a otros países y, principalmente, a causa de los bajos precios de los productos de cuero, provenientes de países asiáticos.

Se pudo establecer que el $58 \%$ de las McEs producen menos de 1.000pieles/mes, mientras que la empresa mediana procesa alrededor de 8.000 pieles/mes, para un total de 20.200pieles/mes aproximadamente, en todo el municipio.

La baja capacidad productiva de las McEs no sólo se demostró por el número de trabajadores contratados, que varía de uno a tres, en el $38,5 \%$ y de siete a diez, en el $46,2 \%$ de los casos; sino también por la baja infraestructura constituida por equipos y maquinaria obsoleta de baja tecnología. La empresa mediana, por su parte, posee una mayor capacidad de producción, representada por un importante número de bombos y de maquinaria de mayor tecnología (Tabla 2).

Se pudo determinar que las curtiembres cerriteñas producen, principalmente, cuero en crosta, tipo nobuck, napa, encerado, graso, marquilla y carnaza, productos que son adquiridos por clientes nacionales, en un $99,8 \%$ y, el 0,2\% restante, es exportado hacia Venezuela. En cuanto al uso final del cuero es comercializado por peleterías y empleado por fabricantes de calzado y de marroquinería; en casos esporádicos es utilizado en la fabricación de muebles.

Tabla 2. Aspectos socioeconómicos de las curtiembres de El Cerrito, en el 2014.

\begin{tabular}{|l|l|c|c|c|c|}
\hline No. & Actividad principal & $\begin{array}{c}\text { Producción } \\
\text { Pieles/mes }\end{array}$ & $\begin{array}{c}\text { No. de } \\
\text { trabajadores }\end{array}$ & No. Bombos & No. Máquinas \\
\hline 1 & Prestación de servicios & 2000 & 8 & 6 & 6 \\
\hline 2 & Recurtido, teñido y acabado & 1500 & 9 & 3 & NS/NR \\
\hline 3 & Proceso completo* & 300 & 7 & 5 & 7 \\
\hline 4 & Proceso completo* & 400 & 3 & 3 & 3 \\
\hline 5 & Proceso completo* & 1000 & 8 & 7 & 9 \\
\hline 6 & Recurtido, teñido y acabado & 150 & 2 & 1 & 0 \\
\hline 7 & $\begin{array}{l}\text { Prestación de servicios y cur- } \\
\text { tición propia }\end{array}$ & 1500 & 10 & 4 & 4 \\
\hline 8 & Proceso completo* & NR & 2 & 2 & 3 \\
\hline 9 & Prestación de servicios & 100 & 1 & 3 & 73 \\
\hline 10 & Proceso completo* & 8000 & 150 & 29 & 6 \\
\hline 11 & Prestación de servicios & 500 & 4 & 6 & 6 \\
\hline 12 & $\begin{array}{l}\text { Prestación de servicios y cur- } \\
\text { tición propia }\end{array}$ & 2000 & 8 & 4 & 5 \\
\hline 13 & $\begin{array}{l}\text { Prestación de servicios y cur- } \\
\text { tición propia }\end{array}$ & 500 & 2 & 7 & \\
\hline
\end{tabular}

*Cuando la curtiembre realiza más del $80 \%$ del proceso de elaboración del cuero. 
Se observó que las curtiembres de El Cerrito han diversificado su actividad, de tal manera que sólo cuatro de ellas realizan los procesos completos dentro de sus instalaciones; las otras McEs, contratan subprocesos en curtiembres, que se han dedicado al alquiler de servicios. En estas curtiembres, se realizan los procesos de pelambre, descarne, dividido, curtido, recurtido, rebajado, templado y planchado, situación que ha favorecido la proliferación de curtidores informales, que no poseen instalaciones propias. Este grupo está compuesto por unos 45 microempresarios, que tienen el conocimiento y la experiencia en el curtido de pieles, pero disponen de bajo capital, de tal manera que para la elaboración del cuero, alquilan la infraestructura de otras curtiembres, compran las materias primas e insumos necesarios y contratan trabajadores por labor a realizar, estableciéndose así una relación laboral informal.

Es evidente la baja calidad del empleo en las McEs, pues los trabajadores entrevistados informaron que no contaban con seguridad social $(p=0,01471)$, que las jornadas laborales se extendían por más de 10h/diarias, en comparación con la mediana empresa $(p=0,01471)$, donde los horarios no sobrepasaban las $8 \mathrm{~h} /$ diarias; de igual manera, reportaron que los implementos de seguridad industrial son muy escasos o nulos ( $p=0,0037)$. En la tabla 3, se resumen las condiciones laborales de las curtiembres cerriteñas.

Se encontró que no hay relación entre las enfermedades que aquejan a los trabajadores y el cargo desempeñado $(p=0,4276)$; sin embargo, algunos de los trabajadores manifestaron que las principales causas de consulta médica se deben a lumbagos, alergias respiratorias y de piel; con frecuencia sufren cortaduras o golpes en los dedos al manipular las pieles; tampoco cuentan con programas de salud ocu- pacional, a diferencia de la mediana empresa $(p=0,00002)$. Prevenir y minimizar este tipo de situaciones conduciría a mejorar las condiciones laborales y a una mayor competitividad del sector, según logros reportados en paises industrializados (COTANCE IndustriAll, 2012).

En cuanto al nivel educativo del personal vinculado a las curtiembres, se observó que el 3,5\% de los trabajadores ha alcanzado formación profesional, técnica, el 6,2\% y secundaria, el 64,3\%. En el caso de los propietarios y directivos de las McEs ha empezado un proceso de relevo generacional, pues los hijos de curtidores fundadores son ahora los gerentes, quienes recibieron educación universitaria, en un 31\% y secundaria, en un $46 \%$; estos datos demuestran un cambio positivo con respecto a la situación descrita por Restrepo et al. (2006), quienes reportaron el predominio de formación primaria en la cadena del cuero, en el Valle del Cauca; sin embargo, la formación en el arte del curtido sigue adquiriéndose de manera empírica. El 96,4\% de los curtidores manifestaron que aprendieron esta labor a través de familiares o amigos, sólo uno de los entrevistados, declaró haber recibido formación en química del cuero, en una universidad europea. A pesar que la capacitación es crucial para una mejor competitividad en cualquier sector productivo, ninguna de las McEs destinan tiempo ni recursos para dicha actividad, encontrándose una fuerte relación entre el tipo de empresa y la capacitación ofrecida a sus trabajadores $(p=0,0002)$. En este sentido, se evidenció que los proveedores de insumos químicos son quienes realizan esta función y son también los encargados de promocionar nuevos productos para la actualización de los procesos de producción.

Esta es una fuerte debilidad detectada en las McEs de El Cerrito, lo que refleja la necesidad de implementar progra-

Tabla 3. Condiciones laborales de los trabajadores de las curtiembres cerriteñas.

\begin{tabular}{|l|l|l|l|l|l|}
\hline \multirow{2}{*}{ Empleador } & \multicolumn{5}{|c|}{ Condiciones laborales } \\
\cline { 2 - 6 } & \multicolumn{1}{|c|}{$\begin{array}{c}\text { Duración del } \\
\text { contrato }\end{array}$} & Jornada laboral & Seguridad social & \multicolumn{1}{|c|}{$\begin{array}{c}\text { Seguridad } \\
\text { industrial }\end{array}$} & Capacitación \\
\hline $\begin{array}{l}\text { Mediana empresa } \\
(7,7 \%)\end{array}$ & $>1$ año & $8 \mathrm{~h} /$ día & $\begin{array}{l}\text { Afiliación } \\
\text { completa }\end{array}$ & Programa vigente & Eventual \\
\hline $\begin{array}{l}\text { Microempresas } \\
\text { tipo 1 (23\%) }\end{array}$ & $6-12$ meses & $8 \mathrm{~h} /$ día & $\begin{array}{l}\text { Afiliación } \\
\text { completa }\end{array}$ & $\begin{array}{l}\text { Delantales y } \\
\text { botas }\end{array}$ & Ninguna \\
\hline $\begin{array}{l}\text { Microempresas } \\
\text { tipo 2 }(69,3 \%)\end{array}$ & $\begin{array}{l}\text { Por labor a } \\
\text { realizar }\end{array}$ & $10-14 \mathrm{~h} /$ día & Sin afiliación & Ninguna & Ninguna \\
\hline $\begin{array}{l}\text { Curtidores } \\
\text { informales }\end{array}$ & $\begin{array}{l}\text { Por labor a } \\
\text { realizar }\end{array}$ & $10-14 \mathrm{~h} /$ día & Sin afiliación & Ninguna & Ninguna \\
\hline
\end{tabular}

Afiliación completa: EPS, ARL, Fondo de pensiones y Cesantías 
mas de capacitación, que permitan la formación profesional y técnica, en aspectos productivos, de seguridad industrial y sistemas de gestión ambiental, entre otros.

En referencia a los aspectos ambientales asociados a las curtiembres, el factor que causa mayor molestia a los vecinos, es el olor desagradable, producido por emisiones atmosféricas provenientes de la descomposición de las pieles y de desechos acumulados en las curtiembres. Otro contaminante atmosférico, es el polvillo, material particulado, proveniente del proceso de esmerilado, que afecta directamente a los trabajadores; a pesar que causa alergias respiratorias, no se ha implementado ningún programa para su tratamiento o prevención.

En cuanto al manejo de los residuos sólidos, en la mayoría de las McEs, los subproductos, como carnazas y recortes, son clasificados y comercializados. Los residuos de las pieles son almacenados en bolsas, costales o tanques, algunos de estos son sometidos a compostaje o son utilizados por otras industrias, en la producción de gomas, chicles, jabones, pegantes y en alimentos y juguetes para caninos; sin embargo, no existe un programa de gestión de residuos sólidos, pues se consideran desechos que deben ser eliminados y gran parte de los residuos sólidos son recolectados por la empresa Proactiva S.A. ESP. Por otra parte, se pudo constatar que la mediana empresa implementó un programa de gestión de residuos sólidos, tendiente a prevenir la generación y la reducción de residuos en la fuente.

En cuanto a la energía eléctrica, este servicio es suministrado por la empresa EPSA ESP y se emplea en la iluminación $y$ en el funcionamiento de la maquinaria y los bombos. Otra fuente de energía es el gas industrial, usado para el calentamiento del agua requerida en las diferentes fases del proceso productivo; una de las McEs ha implementado paneles solares, lo que se ha traducido en ahorro en costos de operación. El consumo promedio de energía mensual por McE estuvo en el rango de 307 a $4.034 \mathrm{kw} / \mathrm{h}$; por su parte la mediana empresa reportó valores entre 80.000 a $110.000 \mathrm{kw} / \mathrm{h}$.

En cuanto a las fuentes de agua, cada curtiembre posee su propio pozo profundo, con autorización de la CVC. En la mayoría de los casos, el agua se considera un recurso inagotable, disponible en cualquier momento y no un insumo para la producción. Se ha estimado que las curtiembres consumen entre 15 y $40 \mathrm{~m}^{3}$ de agua por cada $1.000 \mathrm{~kg}$ de piel procesada (Centro Nacional de Producción Más Limpia, 2004b)

Las curtiembres son reconocidas por la carga contaminante de los efluentes líquidos que son vertidos, en la mayoría de los casos, al alcantarillado o a las fuentes de agua sin tratamiento previo (Faouzi et al. 2013). Los resultados del índice de calidad del agua (ICA), del río Cerrito, ilustran esta situación, según reporte de la Corporación Autónoma Regional del Valle del Cauca (2006), donde se observa que el ICA disminuye a su paso por el municipio. El mismo comportamiento mostró el Î́ndice de Contaminación por Materia Orgánica (ICOMO), que aumentó considerablemente hacia la desembocadura sobre el río Cauca, con un índice de contaminación entre alto y muy alto.

Este comportamiento ha seguido presentándose en el río Cerrito en los últimos años, según resultados obtenidos por la CVC (Tabla 4), antes de la entrada al municipio (E), el agua tenía buena calidad, pero sufrió un fuerte deterioro a su paso por la ciudad (S); se resalta el valor del oxígeno disuelto, que se tornó extremadamente bajo en la última estación. Igual comportamiento presentaron los valores de DBO, DQO, sólidos totales y coliformes fecales, lo que indica la drástica disminución en la calidad del agua.

La contaminación tiene relación con la actividad curtidora del municipio, al considerar que las McEs vierten sus aguas residuales al alcantarillado, únicamente pretratadas por medio de trampas de grasas y sólidos. Estas aguas deberían ser tratadas por la planta de tratamiento de aguas residuales (PTAR) del municipio, pero infortunadamente no se encontró en funcionamiento, por lo cual, los efluentes líquidos de las curtiembres son vertidos directamente al río Cerrito.

Es de anotar que un $25 \%$ de las curtiembres visitadas poseen PTAR completa, compuesta por conductos de separación de efluentes del pelambre, curtido y recurtido, tanques para oxidación de sulfuros, recolección de baños de cromo, de homogenización y precipitación, deshidratadores de lodos y lechos de secado. Otro $50 \%$ posee un sistema incompleto, compuesto por tanques de recolección de aguas residuales y componentes para el tratamiento fisicoquímico, pero sin unidades para reciclaje de cromo y un $25 \%$ posee un sistema incipiente, que consta únicamente de trampas de grasas y de sólidos; sin embargo, ninguna PTAR de las McEs se encontró en operación, solo se halló funcionando la PTAR de la mediana empresa, que trata entre el 70 y $80 \%$ de sus efluentes, que descarga posteriormente al río Cerrito, con autorización de la CVC. Los resultados de esta investigación indican que la situación ha cambiado en comparación con la información presentada por Sanz et al. (2016), quienes reportaron que las curtiembres de El Cerrito, habían incrementado su competitividad y priorizado la recuperación del río Cerrito, mediante programas de PML.

Adicionalmente, solo la mediana empresa ha continuado con la implementación de programas de gestión ambiental, logrando disminuir la aplicación de cromo, desde el 7,0 al $4,8 \%$, obteniendo aguas residuales con concentraciones de cromo entre 1.020 y $1.100 \mathrm{mg} / \mathrm{L}$ que, posteriormente, son diluidas con otros efluentes, bajando así la concentración del 
Tabla 4. Análisis fisicoquímico del agua del río Cerrito, antes de la entrada al municipio (E) y en la desembocadura sobre el río Cauca (S).

\begin{tabular}{|c|c|c|c|c|c|c|c|c|c|}
\hline \multirow{2}{*}{ Parámetro } & \multirow{2}{*}{ Unidad } & \multicolumn{2}{|c|}{ Mayo 2012} & \multicolumn{2}{|c|}{ Agosto 2012} & \multicolumn{2}{|c|}{ Julio 2013} & \multicolumn{2}{|c|}{ Noviembre 2013} \\
\hline & & $\mathrm{E}$ & $S$ & $\mathrm{E}$ & $\mathrm{s}$ & $\mathrm{E}$ & $S$ & $\mathrm{E}$ & $S$ \\
\hline $\mathrm{pH}$ & Unidades & 8,03 & 7,73 & 7,99 & 7,55 & 8,04 & & 7,2 & 7,74 \\
\hline Turbiedad & UNT & 6,0 & 25 & 3,0 & 31 & 2,2 & 2156 & 0,36 & 12,1 \\
\hline $\begin{array}{l}\text { Sólidos } \\
\text { totales }\end{array}$ & $\mathrm{mgST} / \mathrm{L}$ & 74 & 486 & 94 & 471 & 157 & 1344 & 92 & 522 \\
\hline $\begin{array}{l}\text { Oxígeno } \\
\text { disuelto }\end{array}$ & $\mathrm{mgO}_{2} / \mathrm{L}$ & 7,56 & $<0,5$ & 7,96 & $<0,5$ & 7,75 & $<0,5$ & 7,33 & $<0,5$ \\
\hline DBO & $\mathrm{mgO}_{2} / \mathrm{L}$ & $<0,954$ & 22,4 & $<0,954$ & 21,6 & $<0,954$ & 163 & 1,26 & 56,2 \\
\hline DQO & $\mathrm{mgO}_{2} / \mathrm{L}$ & 7,7 & 81,8 & $<5,33$ & 51,3 & $<5,33$ & 383 & $<5,33$ & 150 \\
\hline $\begin{array}{l}\text { Nitrógeno } \\
\text { total }\end{array}$ & $\mathrm{mgN} / \mathrm{L}$ & $<2,08$ & 10,5 & $<2,08$ & 12,4 & $<2,08$ & 47,6 & $<2,08$ & 12,4 \\
\hline $\begin{array}{l}\text { Cromo } \\
\text { total }\end{array}$ & $\mathrm{mgCr} / \mathrm{L}$ & $<0,294$ & 0,316 & -- & -- & $<0,294$ & 0,426 & $<0,294$ & $<0,294$ \\
\hline $\begin{array}{l}\text { Coliformes } \\
\text { fecales }\end{array}$ & $\mathrm{NMP} / 100 \mathrm{~mL}$ & $9,3 \times 10^{2}$ & $1,5 \times 10^{7}$ & $3,6 \times 10^{1}$ & $6,6 \times 10^{6}$ & $9,1 \times 10^{2}$ & $1,1 \times 10^{8}$ & $3,6 \times 10^{2}$ & $2,4 \times 10^{7}$ \\
\hline
\end{tabular}

E. Balneario Las Brisas. Antes de la entrada al municipio.

S. Después del paso del río por el municipio, hacia la desembocadura sobre el río Cauca.

Fuente: Laboratorio Técnico ambiental. Corporación Autónoma Regional del Valle del Cauca (CVC).

metal, a valores alrededor de $28,3 \mathrm{mgCr} / \mathrm{L}$. Concentraciones relativamente bajas, puesto que para este tipo de efluentes se han reportado valores entre 3.000 y $4.325 \mathrm{mgCr} / \mathrm{L}$ (Instituto Cinara \& Universidad del Valle, 2006; llou et al. 2014).

Efluentes con esas bajas concentraciones de cromo no pueden ser reciclados, por lo tanto, las aguas del curtido son tratadas en la planta de tratamiento con los demás residuos líquidos. Estas prácticas han producido beneficios por ahorros en el consumo de materias primas y en el tratamiento previo al reciclaje de cromo; estos hallazgos demuestran la necesidad de complementar la prevención con el control de la contaminación.

Se observó que los programas de PML y de gestión ambiental no han tenido continuidad en las McEs. Los microempresarios consideran que el costo y la complejidad de la tecnología para el tratamiento de los efluentes líquidos, así como el tiempo requerido para el reciclaje del cromo, les resta competitividad al compararlo con quienes no realizan dicho tratamiento. Situación que demuestra la necesidad de nuevos desarrollos tecnológicos, que resulten más asequibles para las McEs.

Se demostró, que la situación actual en las McEs de El Cerrito es de retroceso o estancamiento en el manejo ambiental, a pesar de los esfuerzos realizados en la década pasada, pues el panorama ha cambiado, no sólo por el cierre de curtiembres, que se beneficiaron de las iniciativas propuestas entre 2004 y 2006, sino porque muchos de los administradores actuales no tienen conocimiento del tema o si fueron capacitados en aquella época, no continuaron aplicando los programas de PML y tampoco han implementado nuevas prácticas de mejoramiento ambiental, situación que demuestra la falta de compromiso social y de acompañamiento institucional, necesario en programas que aún son emergentes en este sector industrial.

Los resultados de este estudio permitieron identificar diferencias socioeconómicas y ambientales en las curtiembres de El Cerrito, determinadas por el tipo de empresa, de tal manera que las McEs se han visto afectadas con mayor fuerza que la mediana empresa, por las políticas nacionales y la decadencia del sector del cuero y sus manufacturas, a nivel nacional e internacional. Situación que se evidencia en el decrecimiento del número de empresas activas, la falta de recursos financieros para nuevas inversiones, disminución en la producción, en el número de empleos generados y en la proliferación de curtidores informales, sumado a la falta de conciencia ambiental, de capacitación, de apoyo técnico e institucional. Este sistema productivo, compuesto en Colombia principalmente por McEs, requiere de un mayor acompañamiento, representado en programas integrales, que garanticen su continuidad en el tiempo, con la partici- 
pación de instituciones académicas y gubernamentales, que posibiliten el cumplimiento de la normatividad ambiental y conduzcan a mejorar su competitividad y sostenibilidad.

Agradecimientos: A Elizabeth Patiño y Jazmín Ochoa, por su colaboración en la fase de campo. Financiación: Este estudio fue financiado por la Universidad del Valle. Conflictos de intereses: Los autores manifiestan que no existen conflictos de intereses que pongan en riesgo la validez de los resultados presentados.

\section{BIBLIOGRAFÍA}

1. AKTIVA, 2013. Estudios sectoriales. El cuero y sus manufacturas en Colombia. Disponible desde internet en: http://aktiva.com.co/blog/ Estudios sectoriales/2013/cuero.pdf (con acceso 18/01/14).

2. ALCALDÍA DE EL CERRITO, VALLE DEL CAUCA, 2013. Plan municipal de gestión del riesgo del municipio de El Cerrito. Ed. Alcaldía de El Cerrito. (Colombia). $214 p$.

3. AZOM, M.R.; MAHMUD, K.; YAHYA, S.M.; SONTU, A.; HIMON, S.B. 2012. Environmental impact assessment of tanneries: A case study of Hazaribag in Bangladesh. Int. J. Environm. Sci. Developm. 3(2):152-156.

4. CENTRO DE PROMOCIÓN DE TECNOLOGÍAS SOSTENIBLES. 2009. Mayor productividad y rentabilidad con producción más limpia. Estudio de caso PM-34 Empresa: Curtiembre ARDICCAL. La Paz Bolivia. Disponible desde internet en: http://www.cpts. org/ prodlimp/casosest/ 34ARDICAL .pdf (con acceso $12 / 01 / 2014)$.

5. CENTRO NACIONAL DE PRODUCCIÓN MÁS LIMPIA. 2004a. Proyecto gestión ambiental en la industria de curtiembre en Colombia. Sistema de referenciación ambiental para el sector curtiembre en Colombia, Bogotá D.C. Disponible desde internet en: http:// www. tecnologiaslimpias.org/curtiembres/diagnosticonacional.pdf (con acceso 03/12/2013).

6. CENTRO NACIONAL DE PRODUICCIÓN MÁS LIMPIA, 2004b. Proyecto gestión ambiental en la industria de curtiembre en Colombia. Manual Ambiental Sectorial. Disponible desde internet en: http://www. tecnologiaslimpias.org/curtiembres/manual.pdf (con acceso 03/12/2013.

7. CORPORACIÓN AUTÓNOMA REGIONAL DE CUNDINAMARCA.; UNIVERSIDAD NACIONAL DE COLOM-
BIA. 2009. Capacitación y acompañamiento técnico en producción más limpia al subsector curtiembres en Villapinzón y Chocontá (Cundinamarca). Disponible desde internet en: http://www.idea.unal.edu.co/ SWITCH/ documentos/Cartilla_PML Curtiembres. pdf (con acceso 06/12/2013).

8. CORPORACIÓN AUTÓNOMA REGIONAL DEL VALLE DEL CAUCA. 2006. Línea base ambiental de la calidad de los recursos hídricos superficiales en el Valle del Cauca. Ed. CVC. (Colombia). 75p.

9. COTANCE; INDUSTRIALL. 2012. Informe medioambiental y social. La industria europea del curtido. Ed. COTANCE. (Brucelas). 48p.

10. DEPARTAMENTO ADMINISTRATIVO NACIONAL DE ESTADÍSTICA. 2014. Cuentas trimestrales - Colombia. Producto Interno Bruto ( PIB ) Cuarto trimestre de 2013 y Total anual Introducción. p.78. Disponible desde internet en: http://www.dane.gov.co/files/ investigaciones/ boletines/pib/bol_PIB_IVtrim13.pdf (con acceso 12/04/2014)].

11. FAOUZI, M.; MERZOUKI, M.; BENLEMLIH, M. 2013. Contribution to optimize the biological treatment of synthetic tannery effluent by the sequencing batch reactor. J. Material Environm. Sci. (Moroco). 4(4):532-541.

12. ILOU, I.; SOUABI, S.; DIGUA, K. 2014. Quantification of pollution discharges from tannery wastewater and pollution reduction by pre-treatment station. Int. J. Environm. Sci. Developm. (India). 3(5):1706-1715.

13. INSTITUTO CINARA.; UNIVERSIDAD DEL VALLE. 2006. Sustancias contaminantes de curtiembres en El Cerrito-Valle del Cauca. Ed. Univalle. (Colombia). 11p.

14. JARAMILLO, A.D.; VÁSQUEZ, P.A.; RESTREPO, I. 2005a. CASO EXITOSO Aplicación de Producción más Limpia en las curtiembres de El Cerrito. Disponible desde internet en: www.crpml.org/ descargar. php?id=2433No Title (con acceso 11/03/2013).

15. JARAMILLO, A.D.; VÁSQUEZ, P.A.; RESTREPO, I. 2005b. Potencial de mejoramiento ambiental a través de la implementación de producción más limpia en las curtiembres de El Cerrito. Disponible desde internet en: www.bvsde.paho.org/bvsacd/agua 2003/ cerria. pdf (con acceso 14/06/2013).

16. LOFRANO, G.; MERIÇ, S.; ZENGIN, G.E.; ORHON, D. 2013. Chemical and biological treatment technolo- 
gies for leather tannery chemicals and wastewaters: A review. Sci. Total Environm. (Holanda). 461462:265-281.

\section{MINISTERIO DE AMBIENTE VIVIENDA Y DESARROLLO} TERRITORIAL. 2006. Guia ambiental para la industria y preparado de cueros. Ed. MAVDT. (Colombia). 138p.

18. R CORE TEAM. 2015. R: A language and environment for statistical computing. R Foundation for Statistical Computing. Vienna, Austria. Disponible desde Internet en: https://www.r-project.org/ (con acceso el 11/02/2016).

19. RESTREPO, I.; VÁSQUEZ, P.A.; VALDERRUTÉN, C.; JARAMILLO, A.D.; VIVAS, G.A. 2006. Cadena del cuero en el Valle del Cauca: PML para el mejoramiento de la competitividad. Ed. Feriva S.A. (Colombia). 107p.

20. SANTOS, T.; TOBÓN, C.; TOLOZA, C.; OSORIO, L.C., CASTIBLANCO, C.; TORO, J. 2007. Expected bio- physical , social and economic benefits of Cleaner Production (CP) implementation: A tanner's experience. Disponible desde internet en: http:// www. switchurbanwater.eu/outputs/pdfs/W4_GEN_ PAP_Biophysical_social_economic_benefits_of CP_implementation_Colombia.pdf (con acceso 04/07/2013).

21. SANZ, M.; SIEBEL, M.; AHLERS, R.; GUPTA, J. 2016. New approaches to cleaner production: applying the SASI method to micro-tanneries in Colombia. J. Cleaner Production. (Reino Unido). 112: 963-971.

22. SHAKIR, L.; EJAZ, S.; ASHRAF, M.; QURESHI, N.; ANJUM, A.; ILTAF, I.; JAVEED, A. 2012. Ecotoxicological risks associated with tannery effluent wastewater. Environm. Toxicol. Pharmacol. (Holanda). 34(2):180-191.

Recibido: Octubre 22 de 2015

Aceptado: Julio 8 de 2016

Cómo citar:

Benítez-Campo, N.; Perafán Cabrera, A. 2016. Las curtiembres de El Cerrito (Valle del Cauca): una mirada en torno a su realidad socioeconómica y ambiental. Rev. U.D.C.A Act. \& Div. Cient. 19(2): 457-466. 